\title{
SCHEDULE OF EVENTS
}

1987 ANNUAL MEETING

THE AMERICAN PEDIATRIC SOCIETY AND THE SOCIETY FOR PEDIATRIC RESEARCH

\begin{tabular}{|c|c|c|c|}
\hline $\begin{array}{c}\text { MONDAY } \\
\text { April } 27\end{array}$ & $\begin{array}{c}\text { TUESDAY } \\
\text { April } 28\end{array}$ & $\begin{array}{c}\text { WEDNESDAY } \\
\text { April } 29\end{array}$ & $\begin{array}{c}\text { THURSDAY } \\
\text { April } 30\end{array}$ \\
\hline & \multirow[t]{2}{*}{$\begin{array}{c}\text { 8:00 A.M.-9:00 A.M. } \\
\text { APS BUSINESS MEETING } \\
\text { 9:00 A.M.-12:30 P.M. } \\
\text { SUBSPECIALTY PLATFORM } \\
\text { SESSIONS I } \\
\text { Page 40A } \\
\end{array}$} & \multirow[t]{2}{*}{$\begin{array}{c}\text { 8:00 A.M.-9:00 A.M. } \\
\text { SPR BUSINESS MEETING } \\
\text { 9:00 A.M.-12:30 P.M. } \\
\text { SUBSPECIALTY PLATFORM } \\
\text { SESSIONS II } \\
\text { Page 76A } \\
\end{array}$} & $\begin{array}{c}\text { 8:00 A.M.-10:00 A.M. } \\
\text { SPR PLENARY SESSION } \\
\text { SPR PRESIDENTIAL ADDRESS } \\
\text { YOUNG INVESTIGATOR AWARD } \\
\text { (Grand Ballroom) } \\
\text { Page 112A }\end{array}$ \\
\hline & & & 10:00 A.M.-12:30 P.M. \\
\hline & \multirow{2}{*}{$\begin{array}{c}\text { 2:00 P.M.-4:00 P.M. } \\
\text { APS PLENARY SESSION } \\
\text { APS PRESIDENTIAL ADDRESS } \\
\text { HOWLAND AWARD } \\
\text { (Grand Ballroom) } \\
\text { Page 57A } \\
\end{array}$} & \multirow{5}{*}{$\begin{array}{c}\text { 2:00 P.M.-5:30 P.M. } \\
\text { APS PRESIDENTIAL } \\
\text { STATE-OF-THE-ART } \\
\text { SYMPOSIUM: } \\
\text { AIDS, Special Challenges for } \\
\text { Infants and Children } \\
\text { (Grand Ballroom) } \\
\text { Page 93A } \\
\text { 2:00 P.M.-5:30 P.M. } \\
\text { SUBSPECIALTY PLATFORM } \\
\text { SESSIONS III } \\
\text { Page 94A }\end{array}$} & $\begin{array}{l}\text { POSTER SYMPOSIA \# 7, 8, 9, } 10 \\
\text { (Marina Ballrooms) }\end{array}$ \\
\hline $\begin{array}{l}\text { 3:30 P.M.-6:00 P.M. } \\
\text { POSTER SYMPOSIA \# 1, 2, } 3 \\
\text { (Marina Ballrooms) }\end{array}$ & & & $\begin{array}{c}\text { POSTER SESSION III } \\
\text { EXHIBITS }\end{array}$ \\
\hline Page 20A & \multirow{3}{*}{$\begin{array}{l}\text { 4:00 P.M.-6:30 P.M. } \\
\text { POSTER SYMPOSIA \# 4, 5, } 6 \\
\text { (Marina Ballrooms) } \\
\text { Page 58A } \\
\text { POSTER SESSION II } \\
\text { EXHIBITS } \\
\text { (Exhibit Hall) } \\
\text { Page 64A }\end{array}$} & & $\begin{array}{c}\text { (Exhibit Hall) } \\
\text { Page 121A } \\
\end{array}$ \\
\hline $\begin{array}{l}\text { POSTER SESSION I } \\
\text { EXHIBITS } \\
\text { (Exhibit Hall) } \\
\text { Page 26A }\end{array}$ & & & $\begin{array}{l}\text { 2:00 P.M.-5:00 P.M. } \\
\text { SPR PRESIDENTIAL } \\
\text { STATE-OF-THE-ART } \\
\text { SYMPOSIUM: } \\
\text { Growth and Differentiation } \\
\text { (Grand Ballroom) } \\
\text { Page 133A }\end{array}$ \\
\hline & & & $\begin{array}{c}\text { 2:00 P.M.-5:30 P.M. } \\
\text { SUBSPECIALTY PLATFORM } \\
\text { SESSIONS IV } \\
\text { Page 134A }\end{array}$ \\
\hline
\end{tabular}

